\title{
Automated, parallel mass spectrometry imaging and structural identification of lipids
}

Citation for published version (APA):

Ellis, S. R., Paine, M. R. L., Eijkel, G. B., Pauling, J. K., Husen, P., Jervelund, M. W., Hermansson, M., Ejsing, C. S., \& Heeren, R. M. A. (2018). Automated, parallel mass spectrometry imaging and structural identification of lipids. Nature Methods, 15(7), 515-518 . https://doi.org/10.1038/s41592-018-0010-6

Document status and date:

Published: 01/07/2018

DOI:

10.1038/s41592-018-0010-6

Document Version:

Publisher's PDF, also known as Version of record

Document license:

Taverne

Please check the document version of this publication:

- A submitted manuscript is the version of the article upon submission and before peer-review. There can be important differences between the submitted version and the official published version of record.

People interested in the research are advised to contact the author for the final version of the publication, or visit the DOI to the publisher's website.

- The final author version and the galley proof are versions of the publication after peer review.

- The final published version features the final layout of the paper including the volume, issue and page numbers.

Link to publication

\footnotetext{
General rights rights.

- You may freely distribute the URL identifying the publication in the public portal. please follow below link for the End User Agreement:

www.umlib.nl/taverne-license

Take down policy

If you believe that this document breaches copyright please contact us at:

repository@maastrichtuniversity.nl

providing details and we will investigate your claim.
}

Copyright and moral rights for the publications made accessible in the public portal are retained by the authors and/or other copyright owners and it is a condition of accessing publications that users recognise and abide by the legal requirements associated with these

- Users may download and print one copy of any publication from the public portal for the purpose of private study or research.

- You may not further distribute the material or use it for any profit-making activity or commercial gain

If the publication is distributed under the terms of Article $25 \mathrm{fa}$ of the Dutch Copyright Act, indicated by the "Taverne" license above, 


\title{
Automated, parallel mass spectrometry imaging and structural identification of lipids
}

\author{
Shane R. Ellis $\odot^{1}$, Martin R. L. Paine $\odot^{1}$ ', Gert B. Eijkel $\odot^{1}$, Josch K. Pauling ${ }^{2,5}$, Peter Husen $\odot^{3}$, \\ Mark W. Jervelund ${ }^{2}$, Martin Hermansson ${ }^{2}$, Christer S. Ejsing ${ }^{2,4 \star}$ and Ron M. A. Heeren ${ }^{1 \star}$
}

\begin{abstract}
We report a method that enables automated data-dependent acquisition of lipid tandem mass spectrometry data in parallel with a high-resolution mass spectrometry imaging experiment. The method does not increase the total image acquisition time and is combined with automatic structural assignments. This lipidome-per-pixel approach automatically identified and validated 104 unique molecular lipids and their spatial locations from rat cerebellar tissue.
\end{abstract}

Mass spectrometry imaging (MSI) is used to map the spatial distributions of hundreds of molecules in complex environments such as biological tissues, uniquely enabling scientists to visualize localized biochemical processes. It routinely offers spatial resolutions of $\sim 50 \mu \mathrm{m}$, and recent developments have yielded resolutions of $1.4 \mu \mathrm{m}^{1}$. MSI has many potential applications, including localized tissue and molecular heterogeneity classification, tissue-dependent metabolism studies, and studies of interactions between biomaterial and living systems. The use of MSI to investigate localized biochemistry is, however, hampered by a lack of methods that allow broad, automated structural identification of the many detected molecules. Such capabilities would bring MSI in line with established 'omics' technologies (for example, lipidomics and bottom-up proteomics) and permit an omics experiment for each pixel. In this paper, we report the development of a workflow for MSI-based lipidomics that provides automated resolution of the spatial and compositional complexity of the tissue lipidome $e^{2,3}$.

Coupling of MSI with high-resolution mass analysis (e.g., Fourier transform ion cyclotron resonance and Orbitrap mass spectrometry) provides deeper insight into the biochemical complexity of tissues and cells ${ }^{4-6}$. However, it does not provide structural identification of the detected molecules. For lipidomics, high mass resolution enables confident assignment of lipid classes (e.g., phosphatidylcholine (PC), phosphatidylethanolamine (PE), phosphatidylserine (PS), phosphatidylglycerol (PG), phosphatidylinositol (PI), and phosphatidic acid (PA)) ${ }^{7}$ and lipid species having distinct composites of fatty acyl (FA) moieties (expressed as the combined number of carbons and double bonds in FA chains). The identification of individual FA chains, achievable only with MS/MS, is necessary for a better understanding of lipid biochemistry, which is increasingly recognized as having important roles in different (patho)physiological processes. For example, changes in FA unsaturation and chain length have been observed in various cancers ${ }^{8,9}$, and lipid spatial distributions in tissues are dependent on cell, tissue, and disease type $^{10}$, highlighting the requirement for detailed lipid identification and spatial localization. Current methods to identify lipids observed in an MSI experiment rely on separate workflows for either manual MS/MS or LC-MS/MS analysis of a tissue homogenate (leading to the loss of spatial information), both of which require additional steps that prolong the analysis time. Although automated MS/MS acquisition during MSI has been reported ${ }^{11-13}$, those studies had several limitations, including relatively low mass resolution that left many isobaric molecules and isotopologs unresolved, low precursor coverage, extended acquisition times, wide precursor selection, and no automated interpretation of resulting complex datasets.

Here we present an MSI methodology that overcomes the limitations of these previous approaches and provides automated structural identification of lipids in situ. It uses parallel data-dependent acquisition (DDA)-imaging and automated interpretation of both high-resolution MSI data (mass resolution: 240,000 full-width at half-maximum at $\mathrm{m} / \mathrm{z} 400$ ) and automated MS/MS acquisition of almost every detected nominal $\mathrm{m} / z$ value (Fig. 1 ). The method takes advantage of a newly developed matrix-assisted laser desorption/ ionization (MALDI)-MSI source and a hybrid ion trap-Orbitrap instrument ${ }^{14}$. Full-scan Fourier transform mass spectrometry (FTMS) data are acquired in parallel with ion trap (IT)-MS/MS spectra at adjacent pixel positions via a DDA approach whereby precursor ions are automatically selected on the basis of the current FTMS scan. IT-MS/MS provides two critical advantages over FTMS fragment ion detection (FT-MS/MS): (i) parallel and independent operation of the Orbitrap, and (ii) single-ion sensitivity, critical for fragment ion detection, given the limited number of ions produced from one $20-\mu \mathrm{m}$ sampling position (Supplementary Fig. 1). From a single experiment, DDA-imaging yields a high-resolution MSI dataset, accurate mass measurements of all detected peaks, and structural information pertaining to almost every nominal mass, similar to in-depth lipidomics analysis of tissue extracts by $\mathrm{MS}^{\mathrm{ALL}}$ analysis ${ }^{15}$. Importantly, IT-MS/MS data are acquired automatically without increasing the experimental time compared with that needed for conventional FTMS-MSI operating with equivalent mass resolving power. This analysis yields a tremendous amount of data that must be processed in order for lipids to be visualized and confidently identified. We therefore developed an automated lipid-identification pipeline based on the ALEX $^{123}$ framework ${ }^{15,16}$ to confidently assign lipid structures using both accurate mass and MS/MS data (Methods). Our approach is complementary to existing annotation platforms such as METASPACE, which uses only full-scan MSI data $^{17}$, and the GNPS network for LC-MS/MS data ${ }^{18}$.

'The Maastricht Multimodal Molecular Imaging Institute (M4I), Division of Imaging Mass Spectrometry, Maastricht University, Maastricht, the Netherlands. ${ }^{2}$ Department of Biochemistry and Molecular Biology, Villum Center for Bioanalytical Sciences, University of Southern Denmark, Odense, Denmark. ${ }^{3}$ Department of Physics, Chemistry and Pharmacy, University of Southern Denmark, Odense, Denmark. ${ }^{4}$ Cell Biology and Biophysics Unit, European Molecular Biology Laboratory, Heidelberg, Germany. ${ }^{5}$ Present address: Theoretical Biophysics, Institute of Biology, Humboldt-Universität zu Berlin, Berlin, Germany. *e-mail: cse@bmb.sdu.dk; r.heeren@maastrichtuniversity.nl 
a DDA-imaging acquisition sequence
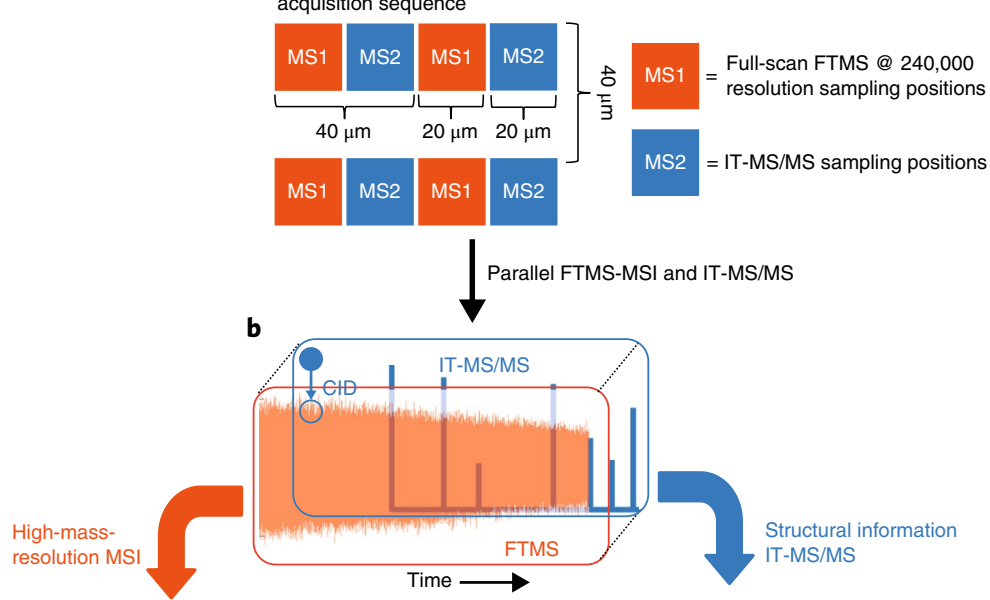

c
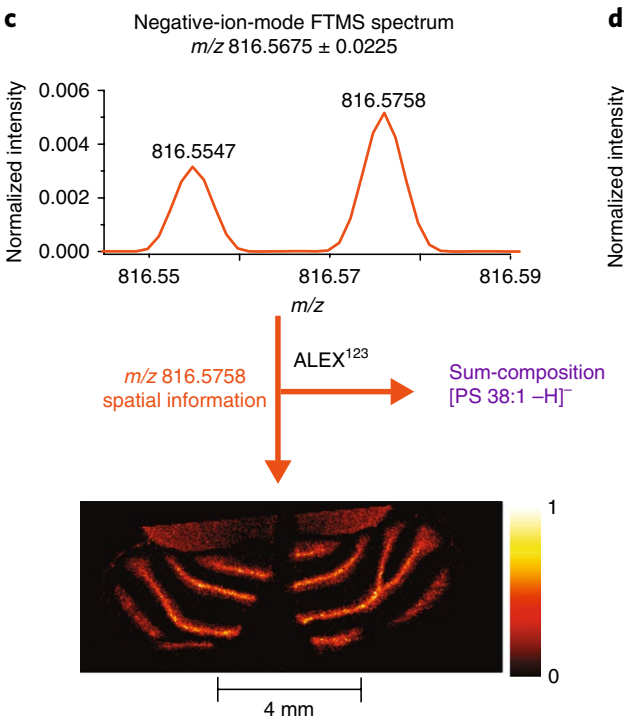

d DDA-imaging IT-MS/MS precursor $\mathrm{m} / \mathrm{z} 816.5 \pm 0.5 \quad-\mathrm{PS}(87)$

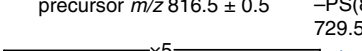

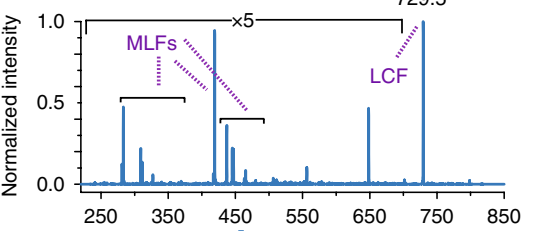

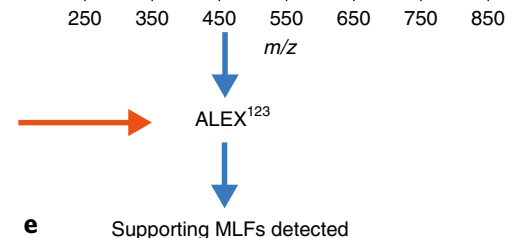
for [PS 38:1-H]

-FA 20:0(+HO) -PS(87) -FA 18:1(+HO) -PS(87)

-FA 20:0(-H) -PS(87) -FA 18:1(-H) -PS(87)

-FA 20:1(+HO) -PS(87) FA 18:0(+O)

-FA 20:1(-H) -PS(87) FA 18:1(+O)

-FA 18:0(+HO) -PS(87) FA 20:0(+O)

$-F A$ 18:0(-H) -PS(87) FA 20:1(+O)

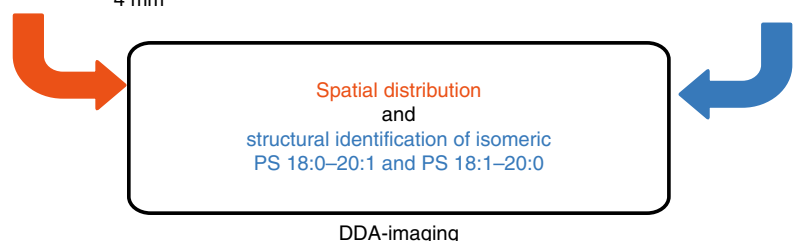

Fig. 1 | Experimental workflow for DDA-imaging and automatic structural identification of detected lipids using ALEX ${ }^{123}$. a,b, Schematic of the acquisition sequence for parallel full-scan FTMS and IT-MS/MS during an MSI experiment (a). At the first position, a high-resolution FTMS spectrum is acquired (orange; example transient in b). During acquisition of the 768-ms transient, the stage moves $20 \mu \mathrm{m}$ horizontally and acquires a data-dependent IT-MS/MS spectrum (blue; example spectrum in b). This sequence is repeated across the tissue surface. c, The FTMS data contain the high-resolution/ accurate mass data for sum-composition assignment of lipids by ALEX ${ }^{123}$, and also the position-resolved spectral data for image reconstruction. In the example spectrum shown, the ion at $m / z 816.5758$ is assigned to [PS 38:1 - H] $]^{-}$by ALEX ${ }^{123}$. d, The parallel IT-MS/MS data provide structural information for molecular identification of lipids. Using the ALEX ${ }^{123}$ database, IT-MS/MS fragment ions matching the sum-composition annotation (from the FTMS spectrum) are assigned. e, In this example, 18:0, 18:1, 20:0, and 20:1 FA-related fragments are identified. IT-MS/MS data and FTMS are then combined (bottom) to generate the molecular identification of $\mathrm{m} / \mathrm{z} 816.5758$, which is assigned to an isomeric mixture of deprotonated PS 18:0-20:1 and PS 18:1-20:0 ions, of which the resulting spatial distribution is also provided by the FTMS data (bottom left image). CID, collision-induced dissociation; MLF, molecular lipid fragment; LCF, lipid class fragment.

We used our method to image lipids in rat cerebellum with a $40-\mu \mathrm{m}$ image pixel size (Fig. 1a). Across an $\sim 10$-h acquisition, $\sim 30,000$ IT-MS/MS and FTMS scans were each automatically acquired, resulting in $>400$ 1-Da-wide MS/MS windows consisting of 10 to $\sim 100$ replicate scans. We carried out DDA-imaging of three cerebellar sections in both positive- and negative-ion modes (full-range $m / z$ spectra are shown in Supplementary Fig. 2). We observed robust and reproducible detection and fragmentation of low-intensity ( $<1 \%$ relative intensity) lipids (Supplementary Figs. 3 and 4). Using the abundant FA-related fragment ions obtained from DDA-imaging in negative-ion mode, we were able to assign FA compositions, thus providing structural information beyond the sum-composition. For example, from three replicate measurements, we were able to assign the negative ion observed at $\mathrm{m} / z 738.5080$ 
a

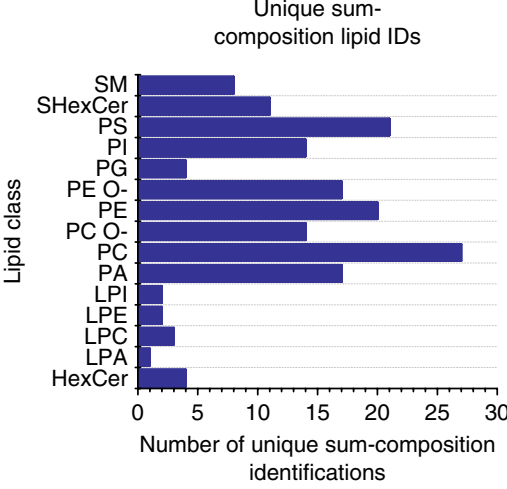

d

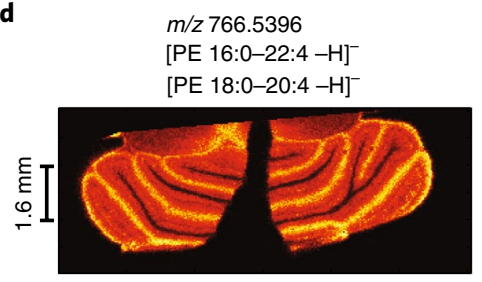

g

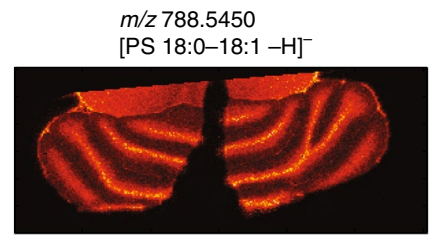

b

Validation of ALEX ${ }^{123}$-identified molecular lipids with FT-MS/MS

Further validated with Automatically identified high-resolution by ALEX ${ }^{123}$ using FT-MS/MS

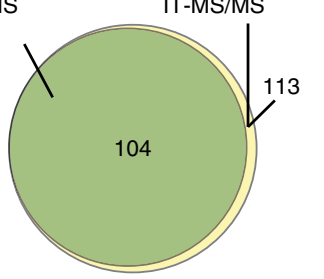

e

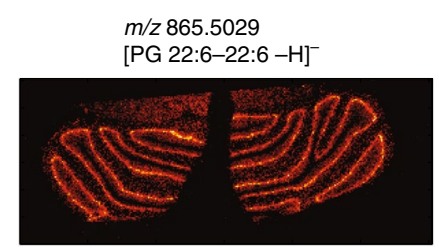

h

$\mathrm{m} / \mathrm{z} 906.6346$

[SHexCer 18:1;2/24:0;1 -H]

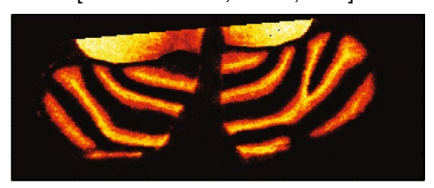

c

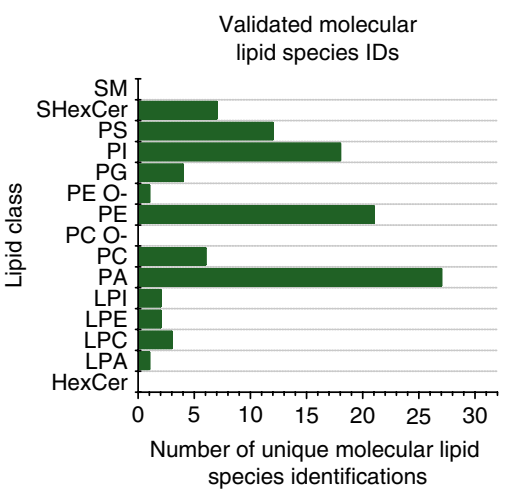

f

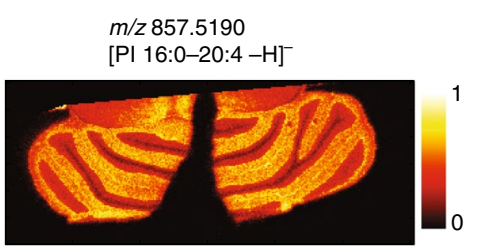

i

Optical image

H\&E-stained tissue
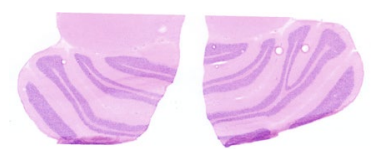

Fig. 2 | Automatic structural annotation of DDA-imaging data. a, The number of unique sum-composition lipid formulas detected by DDA-imaging of rat cerebellum for each lipid class using full-scan FTMS data. All lipids were observed as [M-H]- ions, with the exception of PC/LPC, sphingomyelin (SM), and hexosylceramide (HexCer), which were detected only in positive mode as $[\mathrm{M}+\mathrm{H}]^{+},[\mathrm{M}+\mathrm{Na}]^{+}$, and/or $[\mathrm{M}+\mathrm{K}]^{+}$ions. Sum-composition formulas are assigned within a \pm 2-p.p.m. mass tolerance and with detection in all three technical replicates of at least one polarity (Methods). $\mathbf{b}$, From the parallel IT-MS/MS data, 113 unique molecular lipid species (i.e., identification of lipid class and individual FA moieties) were automatically assigned. Of these, 104 were validated by high-resolution MS/MS. c, The 104 validated molecular lipid species from b, shown by lipid class (Methods). d-h, Example MS images of identified molecular lipids at $m / z 766.5396$ (d), 865.5029 (e), 857.5190 (f), 788.5450 (g), and 906.6346 (h). Each positive- and negative-ion DDAimaging experiment was repeated three times, with similar spectra and images acquired for each technical replicate. The full list of lipid identifications and validations is provided in Supplementary Table 1. i, Optical image of rat cerebellar sections stained with hematoxylin and eosin (H\&E), shown for comparison. We note that it is likely that at least part of the PA lipid population arises from in-source fragmentation of other phospholipid classes. SHexCer, sulfatide; LPE, lysophosphatidylethanolamine; LPC, lysophosphatidylcholine; LPI, lysophosphatidylinositol; LPA, lysophosphatidic acid.

by accurate mass to deprotonated PE 36:4 (Supplementary Fig. 5). Automated and parallel IT-MS/MS data revealed fragment ions suggestive of both palmitic acid (16:0)-containing and arachidonic acid (20:4)-containing PE lipid, that is, the molecular species PE 16:0-20:4, which our spatial FTMS analysis showed to be enriched in the white matter and granule cell layer.

We used an ALEX $^{123}$ automated structural annotation routine for shotgun lipidomics to interpret DDA-imaging data ${ }^{15}$ (Fig. 1). Based on systematic measurements of lipid standards, the ALEX ${ }^{123}$ library includes more than 430,000 molecular lipid species and their expected adduct-specific fragment ions. Lipid species detected by FTMS ( \pm 2 -p.p.m. $m / z$ tolerance) and IT-MS/MS via a lipid classspecific fragment (i.e., $m / z 184$ in positive-ion mode for PC and sphingomyelin lipids) were denoted by sum-composition (e.g., PC 34:1). Molecular lipid species detected by FTMS analysis and IT-MS/MS via FA-specific molecular lipid fragments (e.g., $m / z 281$ for an 18:1 FA) were denoted by molecular composition (e.g., PC 16:0-18:1).

A low-abundance anion at $m / z 816.5758$ localized in the cerebellar white matter was automatically assigned on the basis of FTMS accurate mass to a sum composition of [PS 38:1-H] $]^{-}$(Fig. 1b,c). The FTMS data acquired every $40 \mu \mathrm{m}$ across the tissue revealed lipid spatial distributions. Parallel IT-MS/MS of this nominal mass value revealed an expected lipid class fragment at $\mathrm{m} / z 729.5$ (loss of serine head group) and molecular lipid species fragments suggestive of 18:0-, 18:1-, 20:0-, and 20:1-containing FAs (Fig. 1d,e). Combining both datasets, ALEX $^{123}$ automatically assigned this ion image to the isomeric combination of PS 18:0-20:1 and PS 18:1-20:0, which are enriched in white matter. In the absence of MS/MS, these isomeric contributors would be missed. In cases where characteristic ions were detected in opposite polarities (e.g., PE lipids), we cross-correlated positive- and negative-ion datasets to combine all characteristic fragment ions (Supplementary Fig. 6).

Our method assigned 165 unique sum-composition-level lipid species detected in all three paired positive- and/or negative-ion mode replicates (Fig. 2a and Supplementary Table 1). From these sum-composition lipids, the addition of parallel IT-MS/MS data identified 113 unique molecular lipid species to the individual-FA level. We validated these molecular lipid species by high-resolution FT-MS/MS with extended injection times, which enabled us to unambiguously assign FA neutral loss fragments to individual precursors. We validated 104 of the 113 (92\%) molecular lipid species (Fig. 2b) corresponding to various lipid classes (Fig. 2c), which allowed for assignment of MSI data to both identified lipids 
and anatomical regions (Fig. $2 \mathrm{~d}-\mathrm{i}$ ). This high validation rate illustrates the ability of our method to assign high-confidence identifications on the basis of combined full-scan FTMS and IT-MS/ MS data. For further validation, we compared IT-MS/MS spectra to those obtained from synthetic standards (Supplementary Fig. 7). The full list of identifications, their validated fragment ions, and the overlap across replicates are provided in Supplementary Table 1 and Supplementary Fig. 8. Although LC-MS/MS of a tissue homogenate would probably yield an increased number of identifications, it could not provide the tissue location of identified lipids.

In summary, our method allows automated, high-throughput acquisition of both high-resolution MSI datasets and structurally diagnostic MS/MS data for almost every detected lipid ion without increasing the experiment time. The in-depth MS/MS coverage obtained with a 1-Da isolation window dramatically simplifies spectral interpretation compared with data-independent methods that use wider isolation windows. It thereby overcomes the current limitations of broadband molecular identifications in MSI. Although we applied it to lipids here using the $\operatorname{ALEX}^{123}$ framework, parallel DDA-imaging could be used with alternative identification routines or databases (e.g., LipidBlast ${ }^{19}$ ); be applied to other molecular classes, such as metabolites and peptides, through the use of existing databases and annotation routines; and be applied to alternative analysis or imaging methods. Our workflow allows rapid comparison between different sample types via interactive data visualizations (Supplementary Fig. 9).

This method could be improved in the future through coupling to more efficient desorption/ionization techniques such as laser post-ionization ${ }^{20}$, or derivatization strategies to improve the detection of poorly ionizable lipid classes ${ }^{7}$. This powerful approach should yield insight into localized biochemical pathways and support the construction of region-specific lipidome atlases that facilitate comparisons between local lipid compositions within different tissues. This DDA-imaging approach now brings MSI into the realm of information-rich, shotgun-based omics technologies ${ }^{15}$.

\section{Methods}

Methods, including statements of data availability and any associated accession codes and references, are available at https://doi. org/10.1038/s41592-018-0010-6.

Received: 19 September 2017; Accepted: 28 March 2018; Published online: 21 May 2018

\section{References}

1. Kompauer, M., Heiles, S. \& Spengler, B. Nat. Methods 14, 90-96 (2017).

2. Shevchenko, A. \& Simons, K. Nat. Rev. Mol. Cell Biol. 11, 593-598 (2010).
3. Yetukuri, L., Ekroos, K., Vidal-Puig, A. \& Oresic, M. Mol. Biosyst. 4, 121-127 (2008)

4. Cornett, D. S., Frappier, S. L. \& Caprioli, R. M. Anal. Chem. 80 , 5648-5653 (2008)

5. Smith, D. F., Kilgour, D. P., Konijnenburg, M., O’Connor, P. B. \& Heeren, R M. Anal. Chem. 85, 11180-11184 (2013).

6. Römpp, A. \& Spengler, B. Histochem. Cell Biol. 139, 759-783 (2013).

7. Ryan, E. \& Reid, G. E. Acc. Chem. Res. 49, 1596-1604 (2016)

8. Marien, E. et al. Oncotarget 7, 12582-12597 (2016).

9. Guo, S., Wang, Y., Zhou, D. \& Li, Z. Sci. Rep. 4, 5959 (2014).

10. Zemski Berry, K. A. et al. Chem. Rev. 111, 6491-6512 (2011)

11. OuYang, C., Chen, B. \& Li, L. J. Am. Soc. Mass Spectrom. 26, 1992-2001 (2015)

12. Hansen, R. L. \& Lee, Y. J. J. Am. Soc. Mass Spectrom. 28, 1910-1918 (2017).

13. Perdian, D. C. \& Lee, Y. J. Anal. Chem. 82, 9393-9400 (2010).

14. Belov, M. E. et al. Anal. Chem. 89, 7493-7501 (2017).

15. Almeida, R., Pauling, J. K., Sokol, E., Hannibal-Bach, H. K. \& Ejsing, C. S. J. Am. Soc. Mass Spectrom. 26, 133-148 (2015).

16. Husen, P. et al. PLoS One 8, e79736 (2013).

17. Palmer, A. et al. Nat. Methods 14, 57-60 (2017).

18. Wang, M. et al. Nat. Biotechnol. 34, 828-837 (2016).

19. Kind, T. et al. Nat. Methods 10, 755-758 (2013).

20. Soltwisch, J. et al. Science 348, 211-215 (2015).

\section{Acknowledgements}

This work was supported by the Link program of the Dutch province of Limburg (R.M.A.H.), ITEA and RVO (ITEA151003/ITEA 14001 to R.M.A.H.), the Danish Council for Independent Research | Natural Sciences (DFF - 6108-00493 to C.S.E.), the Lundbeckfonden (R54-A5858 to C.S.E.), the VILLUM Foundation (VKR023439 to C.S.E.), the VILLUM Center for Bioanalytical Sciences (VKR023179 to C.S.E.), Interreg V EMR, and the Netherlands Ministry of Economic Affairs within the "EURLIPIDS" project (S.R.E. and R.M.A.H.). We thank M. Belov (Spectroglyph) and C. Hemedinger (SAS Online Communities) for technical support, and L. Huizing (Maastricht University, Maastricht, the Netherlands) and R. Vreeken (Maastricht University, Maastricht, the Netherlends, and Janssen Pharmaceutica, Beerse, Belgium) for providing intestinal tissue samples from mini pigs.

\section{Author contributions}

S.R.E. and R.M.A.H. conceived the study. S.R.E. and M.R.L.P. performed MSI and MS/ MS experiments. M.R.L.P. prepared the tissue samples. S.R.E., M.R.L.P., and C.S.E. analyzed the data. G.B.E. developed MSI software. J.K.P., P.H., M.W.J., M.H., and C.S.E. developed the ALEX ${ }^{123}$ software and database. S.R.E., C.S.E., and R.M.A.H. wrote the manuscript with input from all other coauthors.

\section{Competing interests}

The authors declare no competing interests.

\section{Additional information}

Supplementary information is available for this paper at https://doi.org/10.1038/ s41592-018-0010-6.

Reprints and permissions information is available at www.nature.com/reprints. Correspondence and requests for materials should be addressed to C.S.E. or R.M.A.H. Publisher's note: Springer Nature remains neutral with regard to jurisdictional claims in published maps and institutional affiliations. 


\section{Methods}

Reagents. Methanol (LC-MS grade), anhydrous chloroform ( $\geq 99 \%$ purity), and crystalline norharmane ( $9 \mathrm{H}-\beta$-carboline) were purchased from Sigma Aldrich (Zwijndrecht, the Netherlands) and used without further purification. Lipid standards were purchased from Avanti Polar Lipids (Alabaster, AL, USA).

Lipid nomenclature. Glycerophospholipid and sphingolipid classes are indicated by their lipid class abbreviations ${ }^{21}$. For lipids identified at the sum-composition level, the combined number of carbons and double bonds in the FA chains is indicated after the lipid class abbreviation. For example, "PE 40:6" denotes a PE lipid with 40 carbons and 6 double bonds spread across both individual FA chains. For lipids identified to the molecular lipid species level (i.e., identification of individual FA chain compositions), individual FAs are indicated in the format of 'total number of carbons:number of double bonds', with individual FAs separated by a dash. For example, "PE 18:0-22:6" indicates a PE lipid containing an 18:0 and a 22:6 FA chain. For ether lipids, ether-bound hydrocarbon chains are preceded with an "O" indicating either 1-O-alkyl ether or 1-O-alkenyl ether (plasmalogen) linkage. For example, PE O-18:1-22:6 is a PE O- lipid with a 22:6 FA chain and an 18-carbon ether-linked chain with one double bond. The double bond could be either that of a 1-O-alkenyl ether or positioned along the remainder of the FA chain as a 1-O-alkyl ether. We note that $s n$ positions and double-bond positions of individual FAs are not defined with this method because of the properties of lowenergy collision-based ion-activation methods.

Molecular lipid fragments (MLFs) were named according to the guidelines described in detail by Pauling et al. ${ }^{22}$. Briefly, MLFs are expressed characteristic of FA chains as either the neutral loss of an FA as an acid (FA(-H)) or as a ketene $(\mathrm{FA}(+\mathrm{HO}))$. For example -FA 20:0 $(-\mathrm{H})$ and -FA 20:0(+HO) indicate the neutral loss of an FA 20:0 moiety as a ketene and fatty acid, respectively. Lipid class fragments were named with the identified lipid class abbreviation and the mass of the characteristic neutral loss or fragment ions. For example, "-PS(87)" indicates the characteristic neutral loss of $87 \mathrm{Da}$ from negative-ion PS lipids, and "PC(184)" indicates the $m / z 184.1$ fragment ion characteristic of PC and sphingomyelin in positive-ion mode. This nomenclature is also used by the ALEX ${ }^{123}$ software framework to denote detected lipid fragment ions and identify lipids at the sumcomposition level and at the molecular lipid species level.

Sample preparation. All animals used in this study were housed in ventilated cages with males and females kept separate, two animals per cage (social housing), and unlimited access to chow and water. Rats (7-week-old Wistar rats) were maintained in the animal facility at Maastricht University and used in accordance with protocols approved by the Animal Care and Use Committee under Animal Experiment Committee (DEC) number 2016-006 AVD107002016720. Rats were killed at 7 weeks of age under general anesthesia (isoflurane). For MALDI imaging experiments, the whole brain was removed immediately after the rat was killed, snap-frozen in liquid nitrogen, and stored at $-80{ }^{\circ} \mathrm{C}$ until sectioned. Transverse rat brain sections $(10 \mu \mathrm{m}$ thick) approximately $4.5 \mathrm{~mm}$ below the bregma point were obtained with a cryo-microtome at $-20^{\circ} \mathrm{C}$ and thaw-mounted on indium tin oxide (ITO)-coated glass slides (Delta Technologies, Loveland, CO, USA).

Mini pig tissue was used in accordance with protocols approved by the Animal Ethics Committee and the Animal Welfare Committee, acting under the Janssen Pharmaceutica N.V. license from the Flemish Authorities Department of Environment, Nature \& Energy, Animal Welfare services, to perform animal experiments (license number LA1100119). A male mini pig (Göttingen minipig (Ellegaard, Denmark)) was killed at 18 months of age under anesthesia (mixture of Zoletil, xylazine, buthorphanol, and ketamine), and its intestine was removed, flushed with saline, frozen on dry ice, and stored at $-80^{\circ} \mathrm{C}$ until sectioning and analysis. Intestinal tissue was sectioned at a thickness of $12 \mu \mathrm{m}$ and thawmounted on ITO-coated glass slides. For positive- and negative-ion lipid imaging, norharmane matrix $(7 \mathrm{mg} / \mathrm{mL})$ in $\mathrm{CHCl}_{3}: \mathrm{MeOH}(7: 3 \mathrm{v} / \mathrm{v})$ was applied to the tissue with a TM-Sprayer (HTX Technologies, USA). Spray conditions were as follows: flow rate, $0.12 \mathrm{~mL} / \mathrm{min} ; \mathrm{N}_{2}$ pressure, 10 p.s.i.; $\mathrm{N}_{2}$ temperature, $30^{\circ} \mathrm{C}$; spray-head velocity, $1,200 \mathrm{~mm} / \mathrm{min}$; track spacing, $3 \mathrm{~mm}$; number of layers, 15 ; drying time between layers, $30 \mathrm{~s}$.

Acquisition of DDA-imaging data. All MSI experiments were performed on an LTQ Orbitrap Elite mass spectrometer (Thermo Fisher Scientific, Bremen, Germany) equipped with an elevated-pressure MALDI ion source incorporating a dual-ion funnel interface (Spectroglyph LLC, Kennewick, WA, USA) as recently described ${ }^{14}$. For MALDI-MSI, the system was equipped with a Q-switched frequency-tripled Nd:YLF laser emitting 349-nm light (Explorer One, Spectra Physics, Mountain View, CA, USA). The laser was operated at a repetition rate of $1 \mathrm{kHz}$ and pulse energy of $\sim 1.2 \mu \mathrm{J}$ as measured by the internal photodiode before any external optics. The laser was focused to a spot $\sim 15 \mu \mathrm{m}$ in diameter as measured by burn patterns in the deposited matrix. The sample region and the entrance to the high-pressure ion funnel were maintained at a pressure of 10.0 $\mathrm{mbar}$, and the exit of the second, low-pressure ion funnel was maintained at 2.0 mbar. Radiofrequency voltages of $620 \mathrm{kHz}, 125 \mathrm{~V}_{\text {0-peak }}$ and $990 \mathrm{kHz}, 80 \mathrm{~V}_{\text {0-peak }}$ were applied to the high- and low-pressure ion funnels, respectively. Extraction of MALDI-generated ions from the slide into the ion funnels was accomplished by an electric field gradient of $\sim 100 \mathrm{~V} \mathrm{~cm}^{-1}$ between the sample holder and the first ion funnel electrode. MALDI stage motion was synchronized with ion accumulation using the LTQ trigger signal indicating commencement of the ion trap injection event. In this way, synchronization with any $\mathrm{MS}^{n}$ event is possible. This trigger signal was accessed via pin 14 of the LTQ J1 connector and activated by setting of the 'injection period' value to 0 in the Tune Plus software (Diagnostics $\rightarrow$ Triggers $\rightarrow$ Injection Period $\rightarrow 0$ ). This trigger signal was then input into a digital delay/pulse generator (DG-535 Stanford Research Systems, Sunnyvale, CA, USA). The delay/ pulse generator outputs a $-4-\mathrm{V}, 100$ - $\mu$ s-wide pulse sent to the trigger input on the MALDI control box that synchronizes stage movement with each scan.

The mass spectrometer was operated with an ion injection time of $250 \mathrm{~ms}$ and automatic gain control turned off. FTMS mass resolution was set to the maximum value of 240,000 (FWHM at $\mathrm{m} / z$ 400) while the linear ion trap was operated at the 'normal' scan speed. All IT-MS/MS spectra were acquired in parallel to the $768-\mathrm{ms}$ Orbitrap transient using collision-induced dissociation with a normalized collision energy of 30 and 40 for the positive- and negative-ion modes, respectively. Because performing MS/MS in a linear ion trap results in an inability to trap fragments below a certain $\mathrm{m} / \mathrm{z}$ value relative to the selected precursor ion, certain diagnostic fragments may not be observed (the so-called low-mass cutoff). The lowest $\mathrm{m} / \mathrm{z}$ fragment that can be detected after mass selection and resonant activation is dependent on the q value of the ion, which in turn is dependent on the voltages applied to the electrodes, the mass and charge of the ion, and the geometry of the ion trap. Under typical operating conditions with a q value of 0.25 for the precursor $\mathrm{m} / \mathrm{z}$ value, fragment ions with $\mathrm{m} / \mathrm{z}$ values approximately less than one-third of the precursor have unstable trajectories and thus are not detected. For lipids, this means the diagnostic $\mathrm{m} / z 184$ ion characteristic of PC and sphingomyelin lipids is not observed. To overcome this limitation in positive-ion mode, we decreased the activation q value from the default value of 0.25 to 0.17 in the Tune Plus software to enable detection of the $m / z 184$ fragment ion.

For all MSI experiments, a stage step size of $20 \mu \mathrm{m}$ (horizontal) $\times 40 \mu \mathrm{m}$ (vertical) was used. Parallel full-scan FTMS (Orbitrap) and IT-MS/MS (ion trap) scans at adjacent $20-\mu \mathrm{m}$ positions were accomplished with an instrument method consisting of two repeating scan events. The first was a 240,000 resolution FTMS scan from $m / z 180$ to $m / z 2,000$. The second was a DDA IT-MS/MS scan, key details of which are summarized in Supplementary Table 2. In this way, one fullscan FTMS and one IT-MS/MS scan were acquired from each $40 \times 40 \mu \mathrm{m}$ sampling region, which is also the pixel size of the MSI data. Both full-scan FTMS and DDA-IT-MS/MS scans were completed at the same time, a key synchronization component of this method. As a result, IT-MS/MS acquisition did not add any time to data acquisitions. MSI experiments were repeated on different tissue sections obtained from the same animal three times each for positive- and negative-ion mode acquisitions. To compare IT-MS/MS sensitivity between FTMS analysis and ion trap fragment detection, we analyzed a cerebellum section under identical conditions with the exception that product ions were analyzed sequentially in the Orbitrap with a nominal mass resolution of 240,000 (FWHM at $m / z$ 400). After acquisition, we evaluated the mass accuracy of several well-known brain lipid species (e.g., [PC 34:1+K] $]^{+}$and [PI 38:4 -H] -). If mass accuracy exceeded 2 p.p.m., the FTMS spectra were internally recalibrated before further processing using the expected signals from [PC 34:1 $+\mathrm{K}]^{+}\left(\mathrm{m} / z\right.$ 798.540964) or [PI 38:4-H] ${ }^{-}$ $(\mathrm{m} / z$ 885.549853) for positive- or negative-ion mode data, respectively.

Validation of molecular lipid species identified by IT-MS/MS with high-massresolution FT-MS/MS. To validate molecular lipid assignments based on IT-MS/MS data, we acquired high-resolution MS/MS data under identical ion-activation conditions using Orbitrap fragment ion detection (mass resolution set to 240,000 at $\mathrm{m} / \mathrm{z} 400$ ). To accumulate high numbers of precursor ions for sensitive detection of fragments, we used a 5,000-ms ion injection time and moved the sample stage continuously across the cerebellum at $1 \mathrm{~mm} \mathrm{~s}^{-1}$. Each $\mathrm{m} / z$ value that was assigned to a molecular lipid species (Supplementary Table 1) was analyzed by this approach. Data were collected for each precursor for $5 \mathrm{~min}$ (negative-ion mode) and $4 \mathrm{~min}$ (positive-ion mode), which resulted in 20 and 25 scans collected, respectively, when accounting for stage flyback time (also fixed at $1 \mathrm{~mm} \mathrm{~s}^{-1}$ ). To validate molecular lipid identifications, we considered only fragments resulting from the direct neutral loss of FA chains from the $[\mathrm{M}+\mathrm{H}]^{+}$or $[\mathrm{M}-$ $\mathrm{H}^{-}$precursors, as only these could be unambiguously assigned to individual precursors with the high mass resolution and accuracy (all molecular lipid species identifications in positive mode used the $[\mathrm{M}+\mathrm{H}]^{+}$ions). This ability to assign individual fragment ions to specific precursors arises as isobaric fragment ions are readily resolved (for example, a 16:0 neutral loss fragment arising from two isobaric precursors), and this enables one to confidently attribute a particular FA neutral loss to a specific and accurate $\mathrm{m} / \mathrm{z}$ value. It thus provides unambiguous confirmation that a certain $\mathrm{FA}$ is present in a given precursor lipid from which it is generated. For each FA chain (excluding ether-linked chains), two fragments are possible corresponding to its loss as either a neutral fatty acid $(\mathrm{FA}(+\mathrm{HO}))$ or a ketene $(\mathrm{FA}(-\mathrm{H}))$. An identification was validated only when all detected fragments were observed within \pm 2 p.p.m. of the theoretical fragment mass and at least two of the possible four fragments with a signal-to-noise ratio greater than 3 were detected. Signal and noise values were taken directly from the Thermo Xcalibur Qual Browser, whereas fragment $m / z$ values were manually collected from the online ALEX ${ }^{123}$ library (http://alex123.info/ALEX123/MS2.php). Some exceptions to this process were as follows: 
1. For PS lipids that did not produce the fragments described above, the classidentifying fragment resulting from loss of the serine headgroup $(-87 \mathrm{Da})$ and the sequential loss of the headgroup and FAs as either a neutral acid or ketene were used. PS lipids were thus validated after detection of the -87-Da fragment ions and two or more fragments resulting from sequential losses of $87 \mathrm{Da}$ and FA chains. As above, all validating fragments were within \pm 2 p.p.m. of their calculated $m / z$ value.

2. For lysophosphatidylethanolamine, lysophosphatidylcholine, lysophosphatidylinositol, and lysophosphatidic acid, only the neutral loss of the single FA as a ketene was observed, and this was used as the validating fragment when within \pm 2 p.p.m. of the calculated fragment $m / z$ value.

3. For sulfatides, only one molecular lipid fragment is used in $\operatorname{ALEX}^{123}$, which is indicative of the $N$-FA chain. This fragment was used for validation when detected within \pm 2 p.p.m. of the calculated fragment $m / z$ value. Detection of this fragment then allowed the nature of the sphinganine/sphingosine moiety to be inferred.

4. For PE ethers (PE O-), because of the potential ambiguity that can arise with the use of fatty acyl neutral losses in negative-ion mode, only characteristic fragments from the $[\mathrm{M}+\mathrm{H}]^{+}$ions were used for validation (a list of adduct ions used for full-scan FTMS and IT-MS/MS confirmation is provided in Supplementary Table 3).

Validation of identified lipids using IT-MS/MS of synthetic standards. Various lipid identifications were also validated by comparison of fragmentation spectra obtained from DDA-imaging experiments to those of synthetic standards corresponding to $\mathrm{ALEX}^{123}$-identified lipids. This validation was done for representative lipid species from six different lipid classes, namely, PI 16:0/16:0, PA 16:0/18:1, PG 16:0/18:1, PS 16:0/18:1, PE 16:0/18:1, and PC 16:0/18:1. Lipid standards (purchased as chloroform solutions) were first diluted to $100 \mu \mathrm{M}$ solutions in $2: 1 \mathrm{CHCl}_{3}: \mathrm{MeOH}(\mathrm{v} / \mathrm{v})$. An aliquot of $10 \mu \mathrm{L}$ of each standard solution was further diluted in $990 \mu \mathrm{L}$ of matrix solution $(7 \mathrm{mg} / \mathrm{mL}$ norharmane in 2:1 $\left.\mathrm{CHCl}_{3}: \mathrm{MeOH}(\mathrm{v} / \mathrm{v})\right)$ to give a final lipid concentration of $1 \mu \mathrm{M}$. This process was repeated separately for each standard. Each lipid:matrix solution $(1 \mu \mathrm{L})$ was then pipetted onto a blank ITO-coated glass slide and analyzed by IT-MS/MS under the same fragmentation conditions used for DDA-imaging experiments (Supplementary Table 2). IT-MS/MS data were acquired for 30-60 s from each standard and the average spectrum used for analysis. For PC, the corresponding $[\mathrm{M}+\mathrm{H}]^{+}$ions were chosen because they provided the MLFs used by ALEX ${ }^{123}$, whereas the $[\mathrm{M}-\mathrm{H}]^{-}$ions were used for all other lipids. These data, along with the corresponding IT-MS-MS data taken from each DDA-imaging technical replicate, are provided in Supplementary Fig. 7. The strong similarity between fragments generated from synthetic standards and those detected and used to make structural assignments validates both the assignments and the ALEX ${ }^{123}$ workflow. Small differences in peak ratios and the presence of additional peaks in the DDA-imaging data are due to the coisolation and fragmentation of isobaric and isomeric ions. Importantly, isobaric ions were resolved during the full-scan FTMS analysis.

Image reconstruction. Custom scripts written in MATLAB (Mathworks, Natick, MA, USA; R2014a) were used to reconstruct MSI data from the full-scan FTMS data. Thermo Fisher .raw data were first converted to profile-mode mzXML format using RawConverter ${ }^{23}$ (http://fields.scripps.edu/rawconv/). The mzXML file contains all scan header information identifying the scan type (full MS or MS/MS), scan number, mass analyzer, and precursor ion mass for MS/MS scans. Every second scan in the DDA-imaging acquisition sequence was a full FTMS scan across each $40 \times 40 \mu \mathrm{m}^{2}$ area of tissue, which was used for image reconstruction. Using the positioning file created by the MALDI control software that relates stage positions to scan numbers, each full MS was then correlated with its acquisition position on the sample ${ }^{14}$. After position assignment, the mean spectrum produced after $\mathrm{m} / z$ binning in 1.5 -mDa increments was used for peak-picking with the PEAPI algorithm ${ }^{24}$. The resulting peak list was then applied to each individual scan to produce the MSI dataset. Individual $\mathrm{m} / \mathrm{z}$ images were then visualized with a MATLAB GUI. All images were generated after total ion current normalization, as is commonplace for MSI data. Image reconstruction and visualization software is provided along with a user guide in the Supplementary Software package.

Automated lipid identification with ALEX ${ }^{123}$. Lipid molecules and fragment ions detected by full-scan FTMS and IT-MS/MS analysis, respectively, were identified by the $\mathrm{ALEX}^{123}$ software, using a curated lipid database with lipid precursor ion $\mathrm{m} / \mathrm{z}$ values (relevant for full-scan FTMS) and structure-specific fragment ion $\mathrm{m} / \mathrm{z}$ values (relevant for IT-MS/MS), as previously reported for shotgun lipidomics data $^{15,22}$. The ALEX ${ }^{123}$ framework uses a curated fragmentation database (based on MS/MS of synthetic lipid standards and features information for more than 430,000 lipid molecules) for searching mass spectral data and a dedicated algorithm for identifying lipid molecules at high confidence ${ }^{22}$. The ALEX ${ }^{123}$ framework is available for download at http://mslipidomics.info/software, together with an example dataset and a user guide (scripts and the user guide are also available from https://git.embl.de/ejsing/ALEX123). Prior to lipid identification, all individual scan filters from each MSI experiment were averaged and saved as text files. Lipid species detected by FTMS were identified using an $m / z$ tolerance of \pm 2 p.p.m., a relative intensity threshold greater than $0.05 \%$ of the most intense lipid peak, corrected for potential ${ }^{13} \mathrm{C}$ isotope interference ${ }^{25}$, and a frequency of 3 (equivalent to being detected in all three tissue technical replicates of a given polarity). Lipid fragment ions detected by IT-MS/MS were identified using an $\mathrm{m} / \mathrm{z}$ tolerance of \pm 0.2 , an absolute intensity threshold of $>0.05$, and a frequency of 3 (equivalent to being detected in all three tissue technical replicates of a given polarity). For high-confidence identification of sum-composition-level lipid species (e.g., PC 34:1) detected by FTMS, at least one confirmatory lipid class fragment detected by IT-MS/MS was required (a list of lipid adduct ions used for identification is presented in Supplementary Table 3). For high-confidence identification of molecular lipid species identified by MLFs, the following criteria were set: (i) the asymmetric molecular lipid species must be detected by at least two complementary pairs of MLFs (except for protonated PE O- species that do not release abundant complementary MLFs); (ii) the molecular lipid species must have an ALEX $^{123}$ score $>0.5$ (calculated as the number of detected MLFs relative to the total number of MLFs available in the $\operatorname{ALEX}^{123}$ database) or an $\operatorname{ALEX}^{123}$ score $\leq 0.5$ but with detection of $>2$ MLFs (with the exception that protonated PE O- species could be detected by at least 2 MLFs); and (iii) confirmation by detection of sumcomposition-level lipid species by FTMS (Supplementary Table 3). We note that molecular lipid species are annotated by molecular composition (e.g., PC 16:0-18:1) where the positioning of FA moieties on the glycerol-phosphate backbone is undefined. This ALEX ${ }^{123}$ lipid identification routine, processing gigabyte mass spectral data files, required 15-20 min per proprietary .raw data file, using a computer running Microsoft Windows 7 Professional (64-bit) with a 2.7 GHz dual core processor and 8 GB RAM.

The raw ALEX ${ }^{123}$ identifications (based on a \pm 0.003 -Da tolerance and before any further filtering based on p.p.m. error, intensity, adduct type, count, MS/MS confirmation, etc. is applied) and the MS/MS data and corresponding fragment assigned for every assigned lipid are provided in the "rat brain_MS1_QC.twbx" and "MSMS visualisation.twbx" files, respectively, in the Supplementary Data. A brief user guide is also provided for the interactive MS/MS file. These files can be opened with the freely available Tableau reader (https://www.tableau.com/ products/reader).

Reporting Summary. Further information on experimental design is available in the Nature Research Reporting Summary linked to this article.

Code availability. Software developed to reconstruct and visualize MSI data from DDA-imaging datasets acquired in Thermo Fisher .raw format is provided, along with a user guide, in the Supplementary Software. ALEX ${ }^{123}$ software for lipid identification, including a user guide with instructions, is available for public download at http://mslipidomics.info/software and https://git.embl.de/ejsing/ ALEX123, and can also be obtained from the corresponding authors upon request.

Data availability. All raw DDA-imaging and MS/MS data are available for download from the Metabolights repository (accession code MTBLS597). Position files (.xml format) for each DDA-imaging acquisition are available in the Supplementary Software.

\section{References}

21. Liebisch, G. et al. J. Lipid Res. 54, 1523-1530 (2013).

22. Pauling, J. K. et al. PLoS One 12, e0188394 (2017).

23. He, L., Diedrich, J., Chu, Y.-Y. \& Yates, J. R. III. Anal. Chem. 87, 11361-11367 (2015).

24. Eijkel, G. B. et al. Surf. Interface Anal. 41, 675-685 (2009).

25. Ejsing, C. S. et al. Anal. Chem. 78, 6202-6214 (2006). 


\section{nature research}

Corresponding author(s): Ron M.A. Heeren

Initial submission $\square$ Revised version

\Final submission

\section{Life Sciences Reporting Summary}

Nature Research wishes to improve the reproducibility of the work that we publish. This form is intended for publication with all accepted life science papers and provides structure for consistency and transparency in reporting. Every life science submission will use this form; some list items might not apply to an individual manuscript, but all fields must be completed for clarity.

For further information on the points included in this form, see Reporting Life Sciences Research. For further information on Nature Research policies, including our data availability policy, see Authors \& Referees and the Editorial Policy Checklist.

\section{- Experimental design}

1. Sample size

Describe how sample size was determined.

\section{Data exclusions}

Describe any data exclusions.

\section{Replication}

Describe whether the experimental findings were reliably reproduced.

\section{Randomization}

Describe how samples/organisms/participants were allocated into experimental groups.

\section{Blinding}

Describe whether the investigators were blinded to group allocation during data collection and/or analysis.
No sample size calculation was performed as the purpose of this work is not to study the biological differences between different animals but to identify the lipid molecules present in a given, single biological tissue. We studied 6 tissue sections of rat brain tissue from the same animal ( 3 in positive and 3 in negative ion mode) with reproducible data acquired for each replicate. Furthermore, during method optimization additional tissue sections were studied and gave reproducible results. Rat brain is the "gold standard" of MSI method development and as such we believe these samples numbers are sufficient for proving the application of our method. We have also studied and provided the results from 6 sections ( $3 x$ positive-ion mode and $3 x$ negative-ion mode replicates) of guinea pig intestine tissue.

No data were excluded

Experimental findings were reliably reproduced

Not relevant. We did not study different groups

Not relevant. We did not study different groups. 
6. Statistical parameters

For all figures and tables that use statistical methods, confirm that the following items are present in relevant figure legends (or in the Methods section if additional space is needed).

$\mathrm{n} / \mathrm{a}$ Confirmed

Х $\square$ The exact sample size $(n)$ for each experimental group/condition, given as a discrete number and unit of measurement (animals, litters, cultures, etc.)

$\square$ A description of how samples were collected, noting whether measurements were taken from distinct samples or whether the same

sample was measured repeatedly

$\bigotimes$ A statement indicating how many times each experiment was replicated

Х

The statistical test(s) used and whether they are one- or two-sided (note: only common tests should be described solely by name; more complex techniques should be described in the Methods section)

$\triangle \square$ A description of any assumptions or corrections, such as an adjustment for multiple comparisons

$\bigotimes \square$ The test results (e.g. $P$ values) given as exact values whenever possible and with confidence intervals noted

Х $\square$ A clear description of statistics including central tendency (e.g. median, mean) and variation (e.g. standard deviation, interquartile range)

Х $\square$ Clearly defined error bars

See the web collection on statistics for biologists for further resources and guidance.

- Software

Policy information about availability of computer code

7. Software

Describe the software used to analyze the data in this study.

MSI data was generated from DDA-imaging datasets using in-house developed software written in MATLAB 2014a. It was adapted from that described in Belov, M.E., Ellis, S.R. et al. Anal. Chem. 89, 7493-7501 (2017). This software is made available with the manuscript

Lipid identification was performed using ALEX123 software that utilized both MS1, MS2 and MS3 data for high confidence lipid identification. A full description and link to the publicly available software is provided with the manuscript.

For manuscripts utilizing custom algorithms or software that are central to the paper but not yet described in the published literature, software must be made available to editors and reviewers upon request. We strongly encourage code deposition in a community repository (e.g. GitHub). Nature Methods guidance for providing algorithms and software for publication provides further information on this topic.

\section{- Materials and reagents}

Policy information about availability of materials

\section{Materials availability}

Indicate whether there are restrictions on availability of unique materials or if these materials are only available for distribution by a for-profit company.

\section{Antibodies}

Describe the antibodies used and how they were validated for use in the system under study (i.e. assay and species).

10. Eukaryotic cell lines

a. State the source of each eukaryotic cell line used.

b. Describe the method of cell line authentication used.

c. Report whether the cell lines were tested for mycoplasma contamination.

d. If any of the cell lines used are listed in the database of commonly misidentified cell lines maintained by $I C L A C$, provide a scientific rationale for their use.
No unique materials were used. All MSI instrumentation used is commercially available

no antibodies were used

no eukaryotic cell lines were used

no eukaryotic cell lines were used

no eukaryotic cell lines were used

no cell lines were used 
Policy information about studies involving animals; when reporting animal research, follow the ARRIVE guidelines

\section{Description of research animals}

Provide details on animals and/or animal-derived materials used in the study.
All rat experiments were performed using Wistar rats acquired from Harlan (Horst, The Netherlands) having weights of 150 grams +/- 10 grams (females) and 250 grams +/- 10 grams (males). Animals were housed in ventilated cages, males and females kept separately, 2 animals per cage (social housing), cage enrichment, unlimited access to chow and water. Animals were euthanized under general anesthesia (Isoflurane), the brain removed and immediately snap frozen and liquid nitrogen and stored at $-80 \mathrm{C}$ until sectioning and analysis.

The 18 month old male Gottingen minipig (Ellegaard, Denmark) weighed $27.9 \mathrm{~kg}$. Minipigs were housed in ventilated cages, males and females kept separately, with a minimal of 2 animals per cage (social housing), cage enrichment, unlimited access to chow and water. Animals were euthanized under anesthesia (mixture of Zoletil ${ }^{\circledR}$, xylazine, buthorphanol and ketamine), the intestine was removed, flushed with saline, frozen on dry ice and stored at $-80^{\circ} \mathrm{C}$ until sectioning and analysis.

Policy information about studies involving human research participants

\section{Description of human research participants}

Describe the covariate-relevant population characteristics of the human research participants. study did not involve human participants 